\title{
Grid diagrams and Khovanov homology
}

\author{
JEAN-MARIE DROZ \\ EMMANUEL WAGNER
}

\begin{abstract}
We explain how to compute the Jones polynomial of a link from one of its grid diagrams and we observe a connection between Bigelow's homological definition of the Jones polynomial and Kauffman's definition of the Jones polynomial. Consequently, we prove that the Maslov grading on the Seidel-Smith symplectic link invariant coincides with the difference between the homological grading on Khovanov homology and the Jones grading on Khovanov homology. We give some evidence for the truth of the Seidel-Smith conjecture.
\end{abstract}

$57 \mathrm{M} 27$

\section{Introduction}

Using symplectic geometry, Seidel and Smith constructed an invariant of oriented links in $S^{3}$ [11]. The Seidel-Smith invariant of an oriented link $L$ is defined as the homology $\mathrm{Kh}_{\text {symp }}^{*}(L)$ of a chain complex associated to $L$. The homological grading of this chain complex is denoted by $P$. They also conjectured that this invariant is isomorphic to the Khovanov link homology $\mathrm{Kh}^{*, *}(L)$ :

Conjecture (Seidel and Smith) For all $k \in \mathbb{Z}$,

$$
\mathrm{Kh}_{\text {symp }}^{k}(L) \cong \bigoplus_{\substack{(i, j) \in \mathbb{Z}^{2} \\ i-j=k}} \mathrm{Kh}^{i, j}(L),
$$

where $i$ is called the Khovanov homological grading and $j$ the quantum grading.

Manolescu showed [5] that the generators of the Seidel-Smith chain complex are in one-to-one correspondence with the intersection points between homology representative arising in Bigelow's construction of the Jones polynomial [1]. Moreover, this correspondence allows us to endow the Seidel-Smith generators with Bigelow's Jones grading $J$. Supporting the Seidel-Smith conjecture, it has been observed by Manolescu that there is, on small examples, enough generators in the Bigelow construction to have 
a complex generated by them whose homology coincides with the Khovanov homology (taking into account the gradings).

Instead of working with an arbitrary plat closure, as is usual when considering Bigelow's construction, we will, in the rest of this article, implicitly restrict ourselves to plat closures that arise from rectangular diagrams. The process by which we associate a plat closure to a rectangular diagram is described at the beginning of Section 2. The purpose of the present article is to introduce a differential on the graded free abelian group generated by the Bigelow intersection points, also called Bigelow's generators. This aim is achieved by proving that there is an injection of Bigelow's generators into enhanced Kauffman states; see Khovanov [4] and Viro [12]. In other words, we will see the Seidel-Smith generators as a subset of the generators of the Khovanov chain complex. Moreover, we prove that, as expected, the gradings verify $P=i-j$ and $j=J$. Our main theorem is:

Theorem 1 There exists a differential $\delta$ on the free abelian group $B$ generated by Bigelow's generators that respects $J$, increases $P$ by 1 and such that the homology of the chain complex $(B, \delta)$ is the Khovanov homology.

Our complex $(B, \delta)$ is homotopic to the original combinatorially defined complex of Khovanov [4]. This gives strong evidence supporting the Seidel-Smith conjecture by generalizing Manolescu's observation. Our main theorem remains true for odd Khovanov homology; see Ozsváth, Rasmussen and Szabó [8] and Theorem 9.

The techniques used below are of intrinsic interest. Namely, we develop a combinatorial description of the Jones polynomial and of Khovanov homology in terms of rectangular diagrams (see Section 1). In particular, our result gives an alternative proof of the equivalence of Bigelow's definition of the Jones polynomial. In addition, grid diagrams appear in the combinatorial description of link Floer homology as in Manolescu, Ozsváth and Sarkar [6]. This new description will be used in future work to investigate the relation between Khovanov type homologies and Heegaard Floer-type homologies (see eg Ozsváth and Szabó [9], Rasmussen [10] and Grigsby and Wehrli [3]).

Plan of the paper In Section 1, we introduce all definitions and notation necessary to compute the Jones polynomial from a grid diagram. In Section 2, we construct an injection of Bigelow's generators into enhanced Kauffman's states. In Section 3, we prove relations between the gradings. Section 4 is devoted to the proof of the main theorem. 
Acknowledgements We would like to thank Anna Beliakova for helpful conversations and pointing us to the paper of Manolescu. The idea of using rectangular diagrams for the Bigelow setting was suggested to us by Anna Beliakova. The present article would not exist without the kind encouragements of Benjamin Audoux. We also thank Oleg Viro and Yuanyuan Bao for pointing out an incomplete argument in a previous version of the paper.

\section{Definitions and notation}

Grid diagrams and links A grid diagram of size $n \in \mathbb{N}-\{0,1\}$ is a $(n \times n)$ - grid whose squares may be decorated by either an $O$ or an $X$ so that each column and each row contains exactly one $O$ and one $X$. The number $n$ is called the complexity of the grid diagram. Following [7], we let $\mathbb{O}$ denote the set of $O$ 's and $\mathbb{X}$ the set of $X$ 's. The $X$ 's and the $O$ 's are called the punctures of the grid diagram.

From any grid diagram, one can construct an oriented link diagram. For this purpose, one should join the $X$ to the $O$ in each column by a vertical segment and the $O$ to the $X$ in each row by an horizontal segment that passes under all the vertical segments. We choose the orientation to be from the $O$ 's to the $X$ 's on the horizontal lines and from the $X$ 's to the $O$ 's on the vertical lines. This produces a planar rectangular diagram for an oriented link in $S^{3}$. Any oriented link in $S^{3}$ admits a planar rectangular diagram [2]. An example is shown in Figure 1.

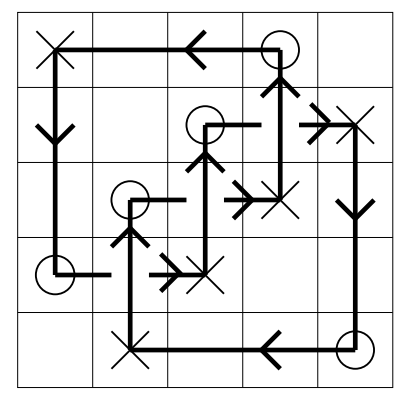

Figure 1: Grid diagram and planar rectangular diagram for the trefoil

The set $\mathcal{G}$ of the Bigelow generators Let $D$ be a planar rectangular diagram of complexity $n$. For each vertical segment, let us draw an oriented curve which winds clockwise around the puncture at the top of the segment and counterclockwise around the puncture at the bottom of the segment. The oriented curves obtained are called figureeights. We choose the figure-eights very narrow and as short as possible. We assume 
that the figure-eights intersect transversally and at most twice the horizontal segments, do not intersect each other and have only one transversal self-intersection. Let $\mathcal{G}$ denote the set of unordered $n$-tuples of intersection points between horizontal segments and (vertical) figure-eights such that each (vertical) figure-eight and each (horizontal) segment contains exactly one point. Let $\mathcal{Z}$ denote the set of intersection points between figure-eights and horizontal segments. We define $\mathbf{x} \in \mathcal{G}(\mathbf{o} \in \mathcal{G}$, respectively) as the set of points of $\mathcal{Z}$ that are nearest to the $X$ 's (the $O$ 's, respectively); see Figure 2 for an example. To each element $g \in \mathcal{G}$, one can associate a unique $n$-tuple, $\bar{g}=\left(\overline{g_{1}}, \ldots, \overline{g_{n}}\right)$ in which $\overline{g_{i}}(i=1, \ldots, n)$ is the $X, O$ or crossing nearest to $g_{i}$.

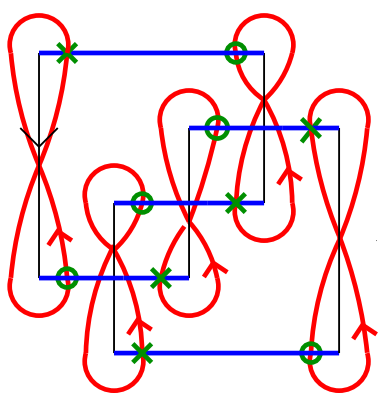

Figure 2: Oriented figure-eights and distinguished elements $\mathbf{x}$ and $\mathbf{o}$ of $\mathcal{G}$

Gradings on $\mathcal{G}$ We will define three gradings on $\mathcal{G}: \bar{P}, T$ and $Q$. For the first grading $\bar{P}: \mathcal{G} \rightarrow \mathbb{Z}$, we orient the figure-eights as in Figure 2. Each $z \in \mathcal{Z}$ is given an integer $p(z):+1$ if the part of the figure-eight on which $z$ sits is oriented upward, 0 otherwise. Given $g=\left(z_{1}, \ldots, z_{n}\right) \in \mathcal{G}, \bar{P}(g)=p\left(z_{1}\right)+\cdots+p\left(z_{n}\right)$. For example, in Figure 2, $\bar{P}(\mathbf{x})=2$ and $\bar{P}(\mathbf{o})=2$.

Given finite sets of points $A$ and $B$ in the real plane, we define $\mathcal{I}(A, B)$ to be the number of pairs $\left(a_{1}, a_{2}\right) \in A$ and $\left(b_{1}, b_{2}\right) \in B$ such that $a_{1}<b_{1}$ and $a_{2}<b_{2}$. The grading $T: \mathcal{G} \rightarrow \mathbb{Z}$ is defined by $T(g)=\mathcal{I}(g, g)$, for $g \in \mathcal{G}$.

We define a relative grading $Q$ on $\mathcal{G}$. Consider two elements $g=\left(g_{1}, \ldots, g_{n}\right)$ and $h=\left(h_{1}, \ldots, h_{n}\right)$ in $\mathcal{G}$. To define the difference $Q(g)-Q(h)$, we consider the loop $\gamma(g, h)$ in the configuration space of $n$ points in $\mathbb{R}^{2}$ defined as follows (see also Bigelow [1]). We start at $g$, go along the horizontal segments to $h$, then go back along the vertical figure-eights to $g$. We can also see $\gamma(g, h)$ as a family of closed immersed oriented curves in $\mathbb{R}^{2}$. Then $Q(g)-Q(h)$ is defined to be the sum of the winding numbers of these closed immersed curves around the $X$ 's and the $O$ 's. In other words, for each $X$ and $O$, we count algebraically the number of times each 
immersed curve goes around the puncture: +1 for each time a curve goes around a puncture counterclockwise and -1 for each time a curve goes around a puncture clockwise and we take the sum over all curves and all punctures as relative grading. We define the absolute grading $Q$ by setting $Q(\mathbf{x})=0$.

Normalization of the gradings and the Jones polynomial We introduce two classical quantities associated to an oriented link diagram $D$ (and hence to an oriented planar rectangular diagram). Given an oriented link diagram $D$, we resolve all the crossings of $D$ as in Figure 3. We obtain a disjoint union of oriented circles embedded in $\mathbb{R}^{2}$.

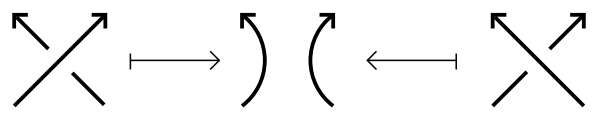

Figure 3: Oriented resolution

We call these circles the Seifert circles of $D$. The rotation number of $D$, denoted by $\operatorname{rot}(D)$, is the sum of the contributions of the circles. The contribution of a Seifert circle is +1 if it is oriented counterclockwise and -1 otherwise. Given a crossing $c$ of an oriented link diagram $D$, we define $w(c)$ as in Figure 4. We define the writhe $w(D)$ of $D$,

$$
w(D)=\sum_{c \text { crossings of } D} w(c) .
$$

Let $n_{+}$be the number of positive crossings and $n_{-}$the number of negative crossings of $D$. We have $w(D)=n_{+}-n_{-}$.

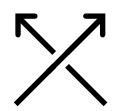

Positive crossing $c$

$w(c)=+1$

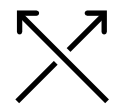

Negative crossing $c$

$$
w(c)=-1
$$

Figure 4: Crossings

We define the Jones grading $J$ and the homological grading $P$. Given a planar rectangular diagram $D$, for an element $g \in \mathcal{G}$, we set

$$
\begin{aligned}
& J(g)=2(T(g)-Q(g))-2 T(\mathbf{x})+\operatorname{rot}(D)+w(D), \\
& P(g)=\bar{P}(g)-\bar{P}(\mathbf{x})-\operatorname{rot}(D)-w(D) .
\end{aligned}
$$


We will show, Corollary 7 below, that the Jones polynomial of an oriented planar rectangular diagram $D$ can be written as

$$
V(D)(q)=\sum_{g \in \mathcal{G}}(-1)^{P(g)} q^{J(g)} .
$$

\section{Bigelow's generators and enhanced Kauffman states}

From plat closures to rectangular diagrams Equation (1) is a simple reformulation of Bigelow's homological definition of the Jones polynomial. In [1], Bigelow computes the Jones polynomial of a link represented as the plat closure of a braid. Our set $\mathcal{G}$ is seen in the setting of Bigelow's definition as the set of intersection points between a cycle in homology represented by the figure-eights and another cycle represented by the horizontal segments.

In [5, Section 3, pages 15-16], Manolescu explains how the plat closure of a braid can be given as a flattened braid diagram. A rectangular diagram can easily be transformed into a flattened braid diagram. Starting with a planar rectangular diagram in the plane with figure-eights drawn, we apply a diffeomorphism of the plane sending all horizontal segments of the rectangular diagram to consecutive nonintersecting segments on a line; see Figure 5. We obtain a flattened braid diagram. Our definitions of the

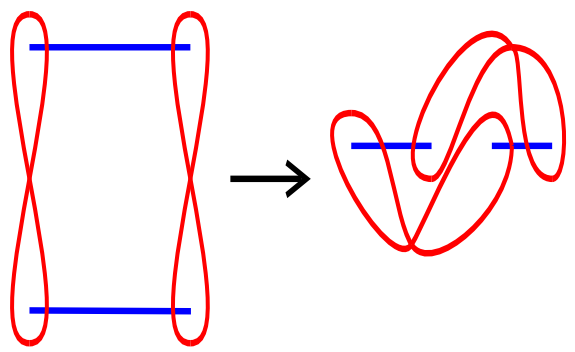

Figure 5: From a rectangular diagram to a braid closure

different gradings are obtained by "pulling back" the gradings originally defined by Bigelow along the diffeomorphism defined above. Notice that we use the notation and normalizations of Manolescu.

The injection $\phi$ from $\mathcal{G}$ to $\mathcal{H}$ Fix an oriented rectangular diagram $D$. We construct a bijection between the set $\mathcal{G}$ and a subset of the enhanced Kauffman states of an oriented link diagram. An enhanced Kauffman state of $D$ is a choice of one resolution 


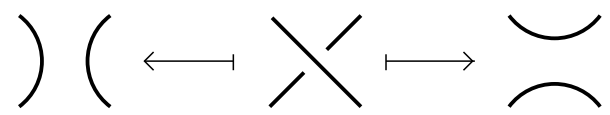

Figure 6: Kauffman resolutions

for each crossing of $D$ (see Figure 6), together with a choice of orientation on every resulting circle; see Figure 7 for an example. We call a choice of resolution for each crossing of $D$ a resolution of $D$. We define $\mathcal{K}$ to be the set of enhanced Kauffman states.

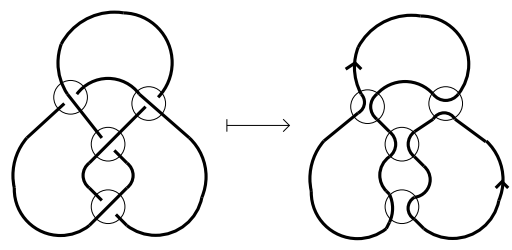

Figure 7: Example of an enhanced Kauffman state

We define $\mathcal{H}$ to be the set of enhanced Kauffman states associated to $D$ such that around each crossing, the arcs coming from the resolutions are oriented as in one of the configurations depicted in Figure 8.

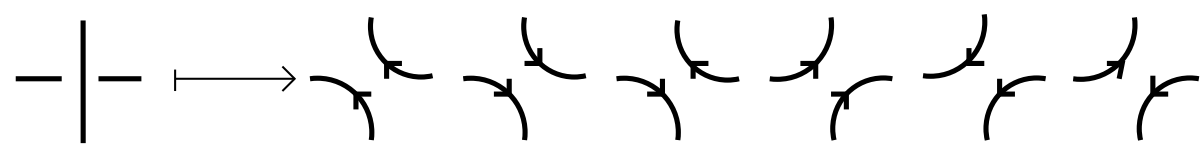

Figure 8: Allowed orientations around a resolution in an enhanced Kauffman state

Notice that the enhanced Kauffman states that are not in $\mathcal{H}$ are those for which at least near one crossing the orientation is as in Figure 9.

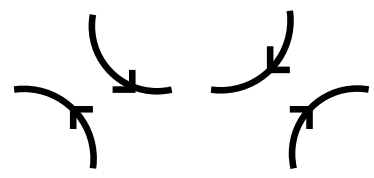

Figure 9: Forbidden orientations around a resolution in an enhanced Kauffman state

Given $g=\left(g_{1}, \ldots, g_{n}\right) \in \mathcal{G}$, we explain how to associate to $g$ an element $\phi(g) \in \mathcal{H}$; see eg Figure 11. Thinking of $D$ as a set of horizontal and vertical segments, we 
can subdivide all the segments in $D$ at the crossings of $D$ to obtain a set of smaller segments that we denote by $\operatorname{seg}(D)$. To an element $g \in \mathcal{G}$ corresponds an orientation of the segments of $\operatorname{seg}(D)$ by the following two rules:

- A vertical segment $s$ is oriented upward when the intersection point of $\bar{g}$ nearest to the line containing $s$ is lower than $s$. It is oriented downward otherwise.

- An horizontal segment $s$ is oriented leftward when the intersection point of $\bar{g}$ nearest to the line containing $s$ is at the left of $s$. It is oriented rightward otherwise.

This means that around an intersection point of $\bar{g}$ the orientation of $\operatorname{seg}(D)$ looks like in Figure 10. See the middle diagram of Figure 11 for an another example.

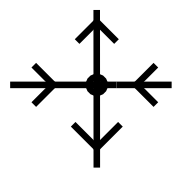

Figure 10: Orientation near a $\overline{g_{i}}$
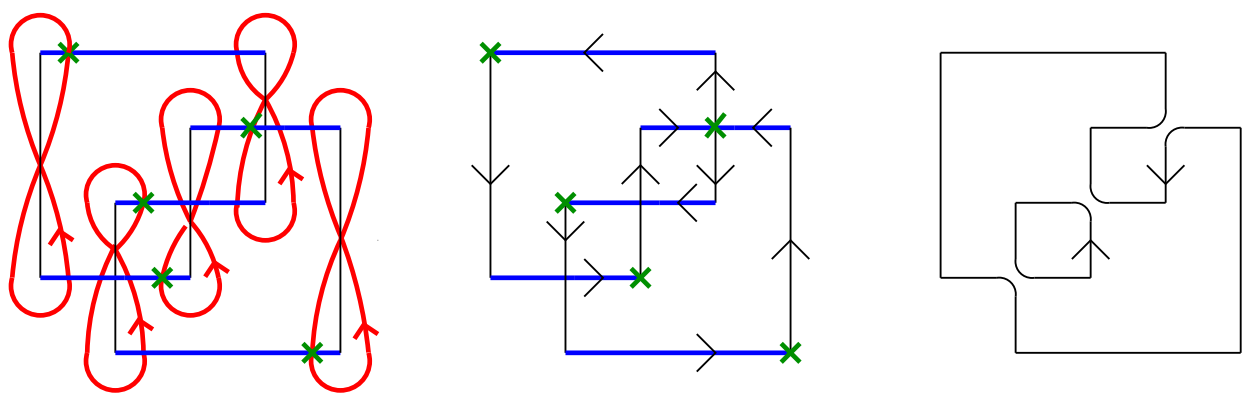

Figure 11: An element of $\mathcal{G}$, the orientation it induces on $\operatorname{seg}(D)$ and its corresponding enhanced Kauffman state $\phi(g)$

We obtain the enhanced Kauffman state $\phi(g)$ from the orientation on $\operatorname{seg}(D)$ by specifying a resolution of each crossing of $D$. The orientation on the circles of $\phi(g)$ being induced in the obvious way by the orientation on $\operatorname{seg}(D)$. For a crossing $c$, if there is no intersection point of $g$ near $c$, we resolve $c$ in the only way that is coherent with the orientation on $\operatorname{seg}(D)$ (see Figure 3). If there is an intersection point $\overline{g_{i}}$ of $\bar{g}$ at $c$, the resolution depends on the position of $g_{i}$. There are four possible cases and Figure 12 describes how to resolve in each case. Since the orientation on $\operatorname{seg}(D)$ is coherent with our choices of resolutions, the function $g \longmapsto \phi(g)$ is well defined. 

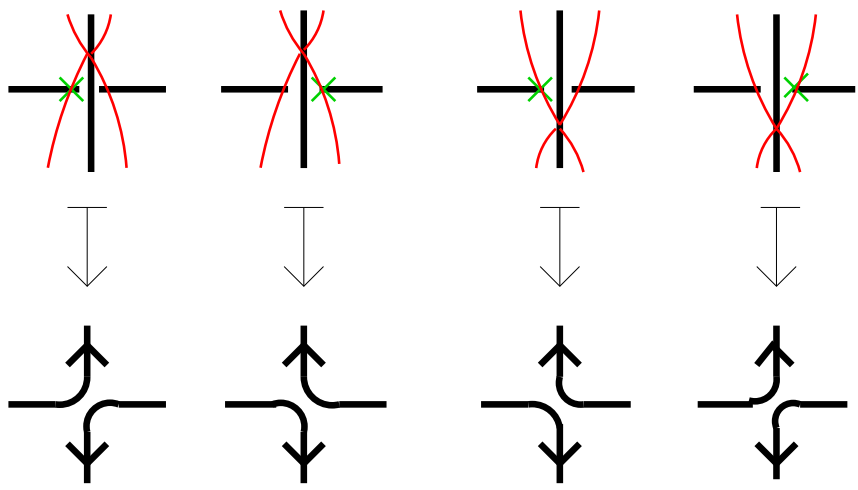

Figure 12: Resolution near a $g_{i}$

Theorem 2 Let $D$ be an oriented planar rectangular diagram. The map $\phi: g \mapsto \phi(g)$ defines a bijection between $\mathcal{G}$ and $\mathcal{H}$.

Proof We define a map $\psi$ from $\mathcal{H}$ to $\mathcal{G}$; see Figure 13 for an example. Given an enhanced Kauffman state $h$ in $\mathcal{H}$, consider the orientation induced by $h$ on $\operatorname{seg}(D)$.
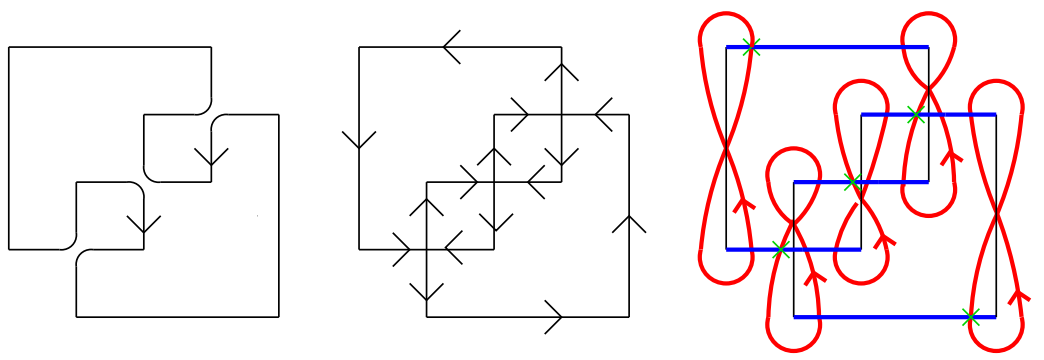

Figure 13: Orientation induced on $D$ by an enhanced Kauffman state and associated element of $\mathcal{G}$

We claim that on a vertical segment of $D$, there is at most one switch of orientation of the kind shown in Figure 14. This follows from the fact that if there were two, then

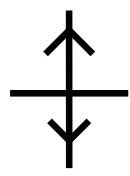

Figure 14: Switch of orientation 
between them, there would be one switch of the type depicted in Figure 15, which is impossible since enhanced Kauffman states in $\mathcal{H}$ do not contain resolutions oriented as in Figure 9. A similar argument proves that, on each horizontal segment, there is at

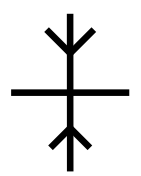

Figure 15: Forbidden switch of orientation

most one switch of orientation (of the allowed type). It follows that one can associate to an element $h \in \mathcal{H}$, an $n$-tuple $\overline{\psi(h)}$ of $X$ 's, $O$ 's or crossings, such that for any $g \in \mathcal{G}, \bar{g}=\overline{\psi(h)}$ implies that $g$ and $h$ induce the same orientation on $\operatorname{seg}(D)$. By using Figure 12, we replace crossings in $\overline{\psi(h)}$ by elements of $\mathcal{Z}$ according to the kind of resolutions around the crossings and the self-intersections of the figure-eights. We replace punctures in $\overline{\psi(h)}$ by their nearest point in $\mathcal{Z}$. We obtain a $n$-tuple $\psi(h) \in \mathcal{G}$. Since clearly $\phi$ and $\psi$ are inverses, $\phi$ defines a bijection between $\mathcal{G}$ and $\mathcal{H}$.

\section{Gradings}

Khovanov homological grading and quantum grading We introduce two gradings $i$ and $j$ on $\mathcal{H}$. By the previous theorem, they induce two gradings on $\mathcal{G}$. Given an element $h \in \mathcal{H}$, consider the underlying resolution $r(h)$ and define $\bar{i}(h)$ to be the number of resolutions in $r(h)$ of the type depicted in Figure 16. Then $i(h)=\bar{i}(h)-n_{-}$. We define $j(h)=\operatorname{rot}(h)+\bar{i}(h)+n_{+}-2 n_{-}$. Notice that $J(\mathbf{x})=j(\mathbf{x})$. As a consequence,

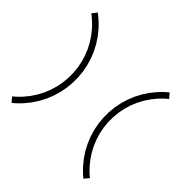

Figure 16: Resolution used in the definition of Khovanov homological grading on $\mathcal{H}$

we define for any $g \in \mathcal{G}$,

$$
\begin{aligned}
j(g) & =j(\phi(g))=\operatorname{rot}(\phi(g))+\bar{i}(\phi(g))+n_{+}-2 n_{-}, \\
i(g) & =i(\phi(g))=\bar{i}(\phi(g))-n_{-} .
\end{aligned}
$$


We express the grading $j(g)$ directly from $g$ described as a set of intersection points. Let us decompose the grading $j$ as a sum of three gradings $j_{1}, j_{2}$ and $j_{3}$.

We define $j_{1}(g)$ to be an algebraic count on the corners of the rectangular diagram. Each corner of the rectangular diagram has a contribution of +1 or -1 . Given $g$, $j_{1}(g)$ is the sum over all corners of these contributions. For the contributions of each corners, see Figure 17 and Figure 18.
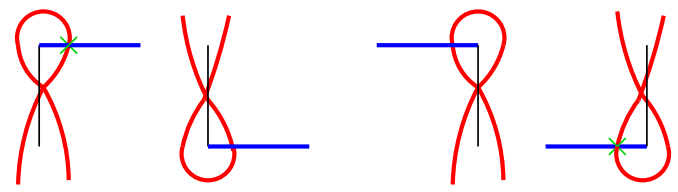

Figure 17: Corners with a contribution to $j_{1}$ of +1
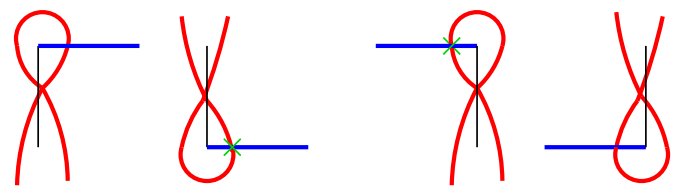

Figure 18: Corners with a contribution to $j_{1}$ of -1

We define $j_{2}(g)$ to be an algebraic count on the crossings that are near an intersection point of $g$. For $g=\left(g_{1}, \ldots, g_{n}\right)$, we consider $\bar{g}=\left(\overline{g_{1}}, \ldots, \overline{g_{n}}\right)$. If $\overline{g_{i}}$ is on a crossing, it has a contribution of +1 or -1 depending on the positions of $g_{i}$ and of the self-intersection of the corresponding figure-eight; see Figure 19 and Figure 20.
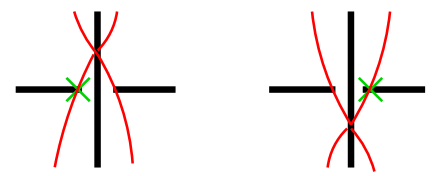

Figure 19: Crossings with a contribution to $j_{2}$ of +1
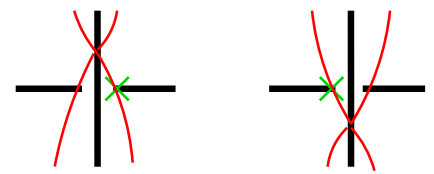

Figure 20: Crossings with a contribution to $j_{2}$ of -1 
We define $j_{3}(g)$ to be an algebraic count on the crossings that do not have a point of $g$ nearby. For $g=\left(g_{1}, \ldots, g_{n}\right)$, consider $\bar{g}=\left(\overline{g_{1}}, \ldots, \overline{g_{n}}\right)$ and consider the crossings where there is no $\overline{g_{i}}$. Each of these crossings has a contribution of +1 or -1 depending on the position of the $g_{i}$ that is on the same figure-eight and on the position of the $g_{j}$ that is on the same horizontal segment; see Figure 21 and Figure 22. Notice that $j_{3}$ does not depend on the position of the self-intersections.
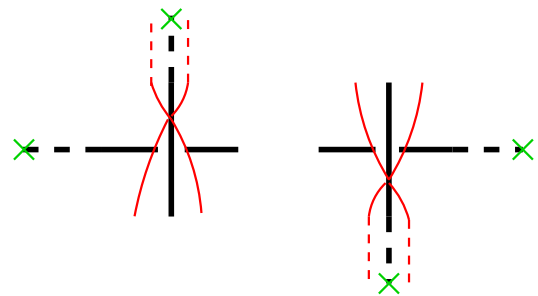

Figure 21: Crossings with a contribution to $j_{3}$ of +1
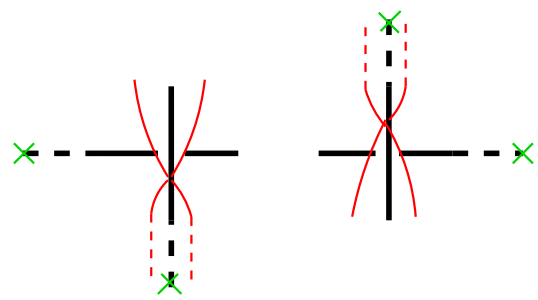

Figure 22: Crossings with a contribution to $j_{3}$ of -1

From previous definitions, we deduce that, for all $g \in \mathcal{G}$,

$$
\begin{gathered}
\operatorname{rot}(\phi(g))=\frac{j_{1}(g)}{4}+\frac{j_{2}(g)}{2}, \\
i(g)=i(\phi(g))=\frac{j_{2}(g)}{2}+\frac{j_{3}(g)}{2}+\frac{n_{+}-n_{-}}{2} .
\end{gathered}
$$

Hence,

$$
j(g)=\frac{j_{1}(g)}{4}+j_{2}(g)+\frac{j_{3}(g)}{2}+\frac{3}{2}\left(n_{+}-n_{-}\right), \quad \text { for all } g \in \mathcal{G} .
$$

Relations between gradings We prove that the gradings verify $P=i-j$ and $J=j$.

Theorem 3 Let $D$ be an oriented planar rectangular diagram. For all $g \in \mathcal{G}$,

$$
P(g)=i(g)-j(g) .
$$


Proof We let $\mathcal{G}^{\prime}$ denote the set of unordered $n$-tuples of intersection points between horizontal segments and vertical figure-eights such that each (vertical) figure-eight contains exactly one point. Observe that $\mathcal{G}$ is a subset of $\mathcal{G}^{\prime}$. Moreover, the gradings $j_{1}, j_{2}$ and $P$ on $\mathcal{G}$ extend in a natural way to $\mathcal{G}^{\prime}$. Since

$$
j(\mathbf{x})=\operatorname{rot}(D)+w(D), \quad i(\mathbf{x})=0 \quad \text { and } \quad P(\mathbf{x})=-\operatorname{rot}(D)-w(D),
$$

we have

$$
P(\mathbf{x})=i(\mathbf{x})-j(\mathbf{x}) .
$$

We prove that $P$ and $i-j$ are equal as relative gradings. Notice that one can go from an element of $\mathcal{G}^{\prime}$ to another by a sequence of elementary moves changing the position of just one intersection point on one figure-eight. It remains to check that $P$ and $i-j$ change by the same amount when such an elementary move is performed. The different cases of elementary moves are presented in Figure 23. Moreover, combining Equations (4) and (5), we obtain

$$
j(g)=\frac{j_{1}(g)}{4}+\frac{j_{2}(g)}{2}+i(g)+w(D), \quad \text { for all } g \in \mathcal{G} .
$$

Thus, we need to prove that $j_{1} / 4+j_{2} / 2$ and $-P$ vary by the same amount when an elementary move is performed. This can be checked directly from the pictures in Figure 23. Note there are a priori 16 other cases to check corresponding to changes of positions of the self-intersections of the figure-eights in the twelve first elementary moves depicted in Figure 23, but these cases follow from the following observation: A change of position of the self-intersection of the figure-eight together with a change of position of the generator by switching side if it sits between the old and the new self-intersections changes neither $j_{1} / 4+j_{2} / 2$ nor $-P$; see Figure 24 .

Corollary 4 For any $g \in \mathcal{G}$, we have the equality

$$
P(g)=-\operatorname{rot}(g)-n_{+}+n_{-} .
$$

Proof Combining Equations (2) and (3) with the previous theorem, we obtain the desired equality.

Theorem 5 Let $D$ be an oriented planar rectangular diagram, for all $g \in \mathcal{G}$,

$$
J(g)=j(g) .
$$

Proof Since

$$
j(\mathbf{x})=\operatorname{rot}(D)+w(D)=J(\mathbf{x}),
$$

it is sufficient to prove that $j$ and $J$ are equal as relative gradings. 

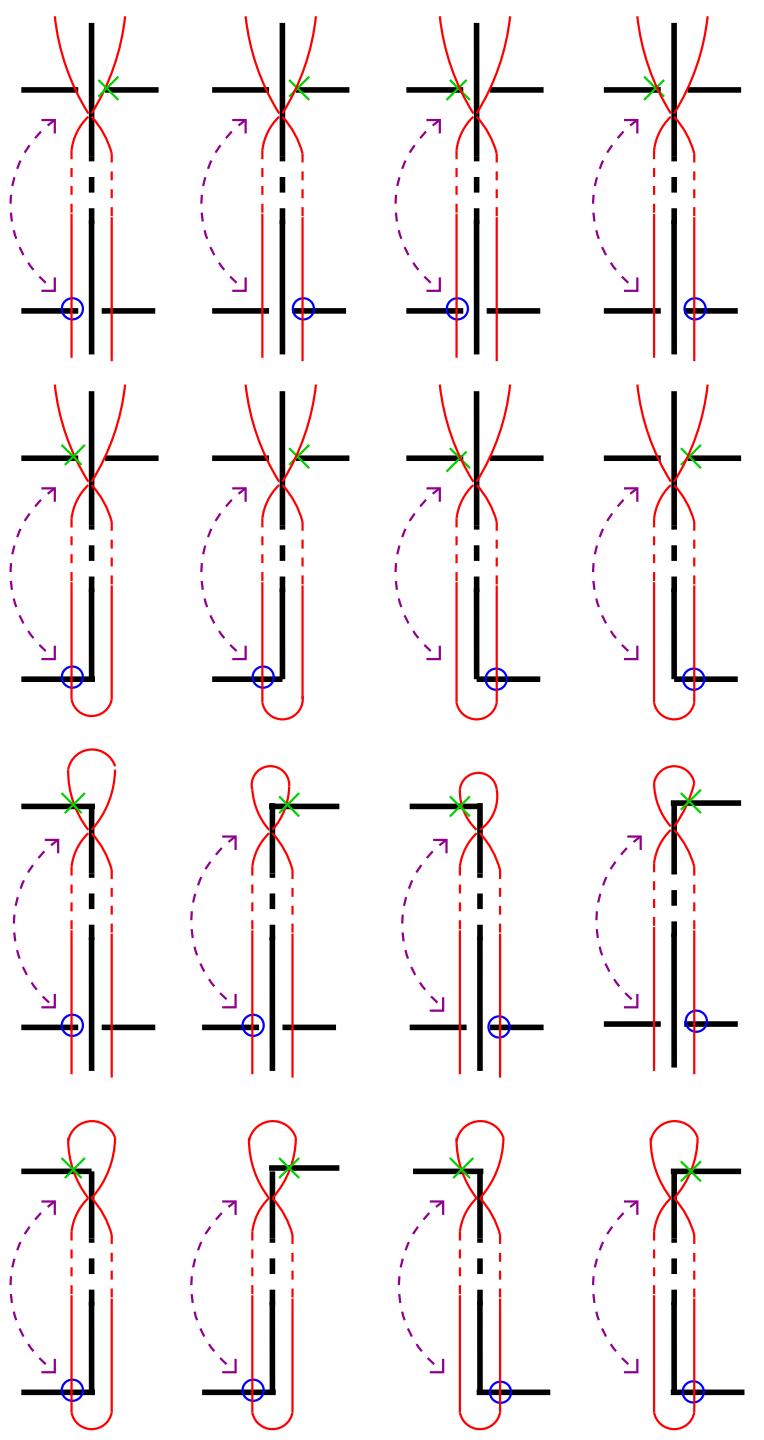

Figure 23: Elementary moves

For $A$ and $B$ two sets of intersection points, we define $\mathcal{I}^{-}(A, B)$ as the number of pairs $\left(a_{1}, a_{2}\right) \in A$ and $\left(b_{1}, b_{2}\right) \in B$ such that $a_{1}<b_{1}$ and $a_{2}>b_{2}$. Given $g=\left(g_{1}, \ldots, g_{n}\right) \in \mathcal{G}$, we define $Q_{\text {far }}(g)$ as follows:

$$
Q_{\mathrm{far}}(g)=\mathcal{I}(g, \mathbb{X} \cup \mathbb{O})+\mathcal{I}(\mathbb{X} \cup \mathbb{O}, g)-\mathcal{I}^{-}(g, \mathbb{X} \cup \mathbb{O})-\mathcal{I}^{-}(\mathbb{X} \cup \mathbb{O}, g) \text {. }
$$



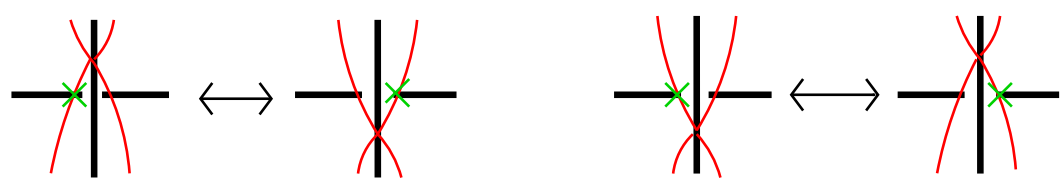

Figure 24: Change of position of a self-intersection point of a figure-eight

We divide any figure-eight in a positive and a negative part by cutting it at its lowest and highest points (Figure 25). We define $Q_{\text {loc }}(g)$ as the number of intersection points

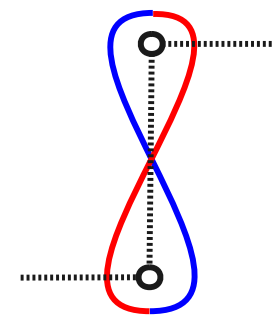

Figure 25: The two parts of the figure-eight

of $g$ on the positive side of the figure-eight (in blue) minus the number of points on the negative side of the figure-eight (in red). We observe that

$$
2 P={ }_{\text {rel }} Q_{\text {loc }} .
$$

(The notation $=_{\text {rel }}$ means that the two sides of the equation are equal up to an additive constant.)

The relative grading $Q$ between two generators $g$ and $h$ is defined as the total winding number of a set of closed paths around the punctures. Let us call $L$ the set of vertical and horizontal half-lines originating from punctures. The winding number of a path can be computed by counting algebraically (with signs) the intersection points of the path with $L$ and dividing by 4 . Using this alternative definition of the winding number and examining separately the contribution of the pieces of the path on horizontal lines and on figure-eights, we check that $4 Q(g)-4 Q(h)=2 Q_{\text {loc }}(g)-2 Q_{\text {loc }}(h)+Q_{\text {far }}(g)-Q_{\text {far }}(h)$, in other words, that, as relative gradings, $4 Q={ }_{\text {rel }} 2 Q_{\text {loc }}+Q_{\text {far }}$.

Let us call good a line either vertical or horizontal that meets two punctures. We call intersection points of good lines good points.

Claim We have the equality of relative gradings:

$$
4 T-j_{3}+\frac{j_{1}}{2}=_{\text {rel }} Q_{\text {far }} .
$$


Proof For each pair of one horizontal good line and one vertical good line, we examine the contributions of all pairs of one puncture or intersection point on the vertical line and one puncture or intersection point on the horizontal line to $4 T, j_{3}, j_{1} / 2$ and $Q_{\text {far }}$. We consider different cases according to where the two good lines intersect. We check that for each pair of good lines, the total contribution to Equation (7) from points on the two lines are independent of the positions of the intersection points of $g$. For example, if the two good lines intersect on a crossing of the rectangular diagram, only $4 T$ and $j_{3}$ are affected and the contribution of the intersection points to $4 T$ and $j_{3}$ cancel. Since each puncture or intersection point appears once on a vertical good line and once on an horizontal good line, taking the sum over all possible pairs of good lines gives twice Equation (7).

Using Equation (7) divided by two and the definition of $j$ by Equation (5), we get

$$
j={ }_{\text {rel }} \frac{j_{1}}{4}+j_{2}+2 T-\frac{Q_{\text {far }}}{2}+\frac{j_{1}}{4} .
$$

Using Equation (6) and the formulation of $P$ in terms of $j_{1}$ and $j_{2}$, we have

$$
-Q_{\text {loc }}={ }_{\text {rel }} \frac{j_{1}}{2}+j_{2} \text {. }
$$

Combining the last two equations gives

$$
j={ }_{\text {rel }} 2 T-\frac{Q_{\text {far }}}{2}-Q_{\text {loc }}
$$

Since $Q_{\text {far }} / 2+Q_{\text {loc }}={ }_{\text {rel }} 2 Q$ and $J==_{\text {rel }} 2(T-Q)$, we deduce $j={ }_{\text {rel }} J$ from which $j=J$ follows.

\section{Relation with Khovanov homology}

In [5], Manolescu noticed that, in the case of the trefoil, a free abelian group whose generators are labeled by Bigelow's generators and are graded according to the grading of their label, has ranks in the different gradings compatible with having as homology the Khovanov homology. We prove that this observation holds for any link. For this purpose, we start with the Khovanov chain complex, ie the chain complex whose generators are enhanced Kauffman states $\mathcal{K}$ and cancel all generators lying in $\mathcal{K} \backslash \mathcal{H}$. We end up with a chain complex homotopic to the original one, with a set of generators in one-to-one correspondence with $\mathcal{G}$. This reduction can be done canonically over $\mathbb{Q}$. Over $\mathbb{Z}$ the reduction seems to depend on some arbitrary choices.

Our reduction over $\mathbb{Q}$ to a smaller complex is canonical in the following sense. Two oriented rectangular diagrams that are sent to each other by diffeomorphisms of the 
plane that send horizontal segments to horizontal segments have isomorphic chain complexes. This implies that the chain complex generated by Bigelow's intersection points is computable from the ambient isotopy type of the flattened braid diagram and is as such a candidate for geometric interpretation.

Proof of Theorem 1 We review a few facts about Khovanov homology (for precise definitions and more $[4 ; 12])$. The Khovanov chain complex is a bigraded complex

$$
C_{\mathrm{Kh}}=\bigoplus_{i, j} C_{\mathrm{Kh}}^{i, j}
$$

with generators associated to enhanced Kauffman states

$$
C_{\mathrm{Kh}}^{i, j}=\bigoplus_{\substack{s \in \mathcal{K} \\ i(s)=i, j(s)=j}} \mathbb{Z} \cdot s .
$$

Observe that $C_{\mathrm{Kh}}^{i}=\bigoplus_{j \in \mathbb{Z}} C_{\mathrm{Kh}}^{i, j}$ is generated by all enhanced Kauffman states obtained by orienting the circles in a resolution $r$ such that $i(r)=i$. Hence, as usual, $C_{\mathrm{Kh}}^{i}$ can be seen as $\bigoplus_{r, i(r)=i} V^{\otimes k(r)}\left\{i+n_{+}-n_{-}\right\}$where $V$ is the two dimensional graded $\mathbb{Z}$-module spanned by 1 and $x$ with $j(1)=1$ and $j(x)=-1, k(r)$ is the number of circles in the resolution $r$ and $\{\cdot\}$ is the shift operator in quantum grading. One can identify an element in $V(r)=V^{\otimes k(r)}$ with a choice of orientations of the $k(r)$ circles. A circle oriented counterclockwise corresponds to a 1 and a circle oriented clockwise corresponds to an $x$. Given a diagram $D$, with $k$ crossings there are $2^{k}$ resolutions of $D$. One can see these resolutions as lying on the vertices of an hypercube of dimension $k$. Hence, the vector spaces $V(r)$ are indexed by the vertices of this hypercube. Similarly, each enhanced Kauffman state sits on a vertex of the hypercube. Two enhanced Kauffman states are connected by the differential of Khovanov homology if and only if they are on both ends of an edge of the hypercube and look around a crossing like one of the 18 pairs in Figure 26. Locally, all possible nonzero differentials are depicted in Figure 26. Naturally, one would have to introduce signs to fully specify the differential $[4 ; 12]$.

We define a new grading $R$ on the Khovanov chain complex as follows. Given an oriented link diagram $D \in \mathbb{R}^{2}$, consider the underlying oriented 4-valent graph $\Gamma$ in which each crossing of $D$ is replaced by a 4 -valent vertex. Choose a point in each connected component of $\mathbb{R}^{2} \backslash \Gamma$. This produces a family of points $\left(x_{i}\right)_{i \in I}$, where $I$ is a finite set. For an enhanced Kauffman state $s$, we define $R(s)$ to be the winding number of the oriented circles of $s$ around the $x_{i}$ 's. In Figure 26, the blue cross and the green cross correspond to $x_{i}$ 's. 
The six differentials in the first column of Figure 26 are connecting generators belonging to $\mathcal{K} \backslash \mathcal{H}$. These differentials respect the grading $R$. In addition, the remaining twelve other types of differentials strictly decrease $R$. Therefore, the increasing filtration associated to the grading $R$ is respected by the differential of the Khovanov complex.
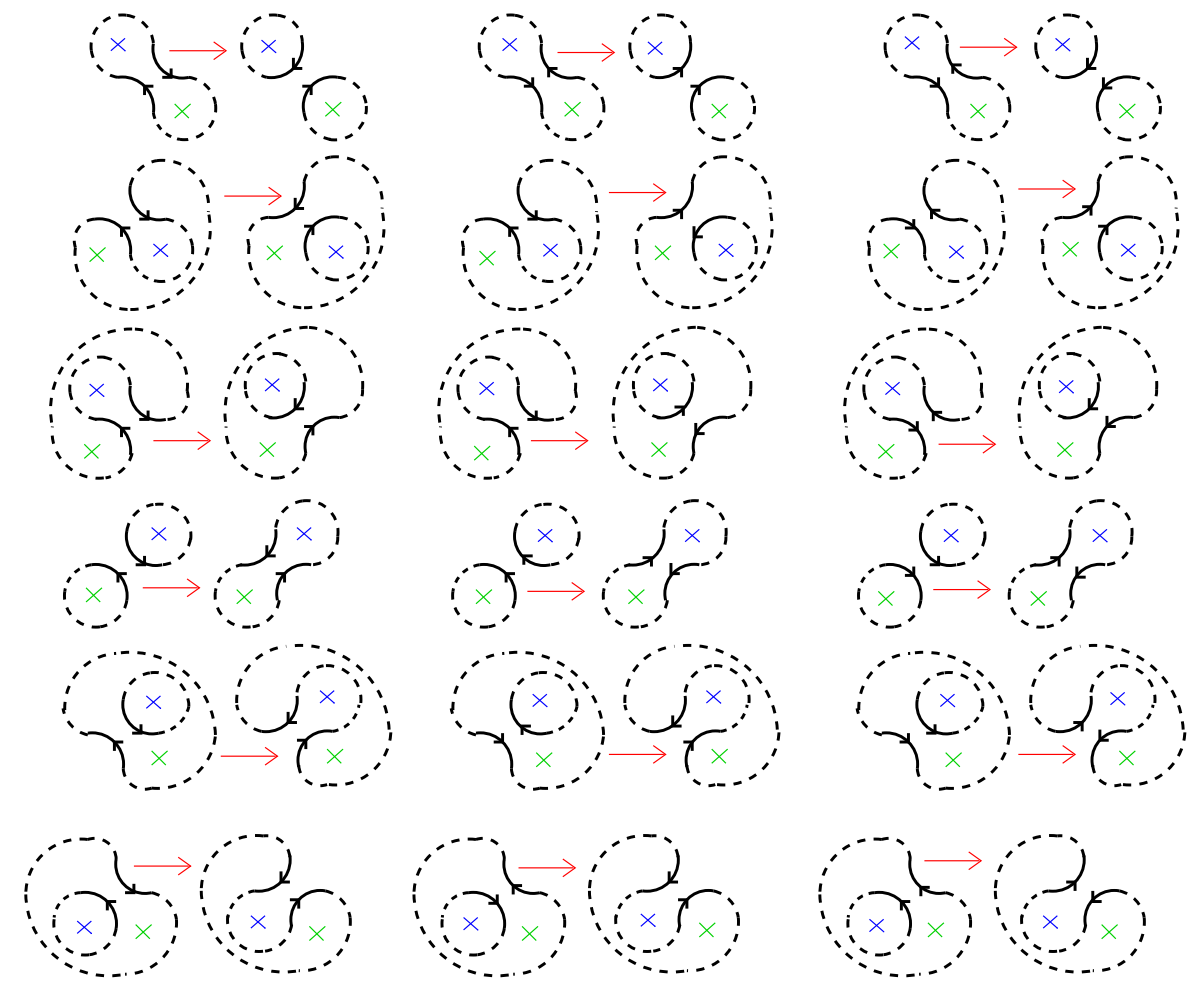

Figure 26: Nonzero differentials in Khovanov complex

We state a lemma in homological algebra generalizing Gaussian elimination. The lemma is proved in the Appendix.

Lemma 6 Given a chain complex $C=\bigoplus_{i \in \mathbb{Z}} C^{i}, \delta: C \rightarrow C$ of degree +1 such that $C=A \oplus B$ as graded abelian group and

$$
\delta=\left(\begin{array}{ll}
a & d \\
c & b
\end{array}\right) \quad \text { with } A \stackrel{a}{\longrightarrow} A, B \stackrel{d}{\longrightarrow} A, A \stackrel{c}{\longrightarrow} B \text { and } B \stackrel{b}{\longrightarrow} B,
$$

and suppose that $(B, b)$ is a chain complex homotopic to zero. Then, for $h: B \longrightarrow B$ of degree -1 such that $-\mathrm{Id}=h b+b h$, the chain complex $(C, \delta)$ is homotopic to

$$
(A, a+d h c) .
$$


Theorem 1 follows from Theorem 3, Theorem 5 and repeated application of Lemma 6. More precisely, let $\left(C_{\mathrm{Kh}}, \delta_{\mathrm{Kh}}\right)$ be the Khovanov chain complex. Let $H$ be the free abelian group generated by $\mathcal{H}$. Consider $B_{0}$ the free abelian group generated by $\mathcal{K} \backslash \mathcal{H}$ and $b_{0}$ the restriction of $\delta_{\mathrm{Kh}}$ to $B_{0}$. We define $i_{\min }=\min \{R(g) \mid g \in \mathcal{K} \backslash \mathcal{H}\}$ and $i_{\max }=\max \{R(g) \mid g \in \mathcal{K} \backslash \mathcal{H}\}$. Then, $B_{0}$ decomposes along the $R$ grading:

$$
B_{0}=\bigoplus_{i=i_{\min }}^{i_{\max }} B^{i}
$$

where $B^{i}$ is the free abelian group generated by $\{g \in \mathcal{K} \backslash \mathcal{H} \mid R(g)=i\}$. The Khovanov differential restricted to $B^{i_{\min }}$ respects the grading $R$ and the restriction $b_{\min }$ is a differential on $B^{i_{\text {min }}}$. Moreover, $\left(B^{i_{\text {min }}}, b_{\text {min }}\right)$ is homotopic to zero. This follows from the fact that this chain complex is a shifted direct sum of hypercube chain complexes, where each hypercube chain complex is obtained by the usual procedure of flattening an hypercube [9, page 17] and replacing every vertex by a copy of $\mathbb{Z}$ and every arrow by + Id or - Id. Each of those hypercube chain complexes is clearly homotopic to zero.

We let $B_{k}$ denote $\bigoplus_{i=i_{\min }+k}^{i_{\max }} B^{i}$. Lemma 6 enables us to defines a differential $\delta_{1}$ on $B_{1} \oplus H$ giving a complex homotopic to $\left(C_{\mathrm{Kh}}, \delta_{\mathrm{Kh}}\right)$. On $B_{1}$, the differential resulting from the application of Lemma 6 is the restriction of $b_{0}$ to $B_{1}$. This can be seen by considering the $R$-grading of the summands of the differential in (8). By iterating this "elimination" of the generators of minimal $R$-grading, one constructs a sequence of complexes $\left(B_{k} \oplus H, \delta_{k}\right)$ for $k \in\left\{0, \ldots, i_{\max }-i_{\min }+1\right\}$. Those complexes are homotopic to each other. The first complex in the sequence is the Khovanov complex and the last complex is generated by $\mathcal{H}$.

Corollary 7 (Bigelow [1]) Given an oriented rectangular diagram $D$,

$$
V(D)(q)=\sum_{g \in \mathcal{G}}(-1)^{P(g)} q^{J(g)}
$$

is the Jones polynomial.

Corollary 8 There exists a differential $\delta$ on the $\mathbb{Q}$-vector space $B$ generated by Bigelow's generators $\mathcal{G}$, that respects $J$, increases $P$ by 1 and such that the homology of the chain complex $(B, \delta)$ is the Khovanov homology. Moreover, over $\mathbb{Q}$, this differential is canonical.

Proof The first part of the statement follows from Theorem 1. The second part follows from the existence, over $\mathbb{Q}$, of canonical homotopies for the applications of 
Lemma 6. We construct these homotopies. In the repeated applications of Lemma 6, the null-homotopic chain complex $B^{\prime}$ is always a shifted direct sum of hypercube chain complexes, where each hypercube chain complex is obtained by the usual procedure of flattening an hypercube and, in this case, replacing every vertex by a copy of $\mathbb{Q}$ and every arrow by $+\mathrm{Id}$ or $-\mathrm{Id}$. As explained before, those hypercubes are null homotopic. In particular, given a decomposition of an hypercube of dimension $n$ into two hypercubes of dimension $n-1$, one can take as homotopy minus the inverse of the differentials between the two hypercubes of dimension $n-1$. Since there are $n$ ways to split an hypercube of dimension $n$ into two hypercubes of dimension $n-1$, there are $n$ homotopies of this kind. We denote them by $h_{1}, h_{2}, \ldots, h_{n}$. Each of them comes from a choice of splitting. We consider

$$
h=\frac{1}{n} \sum_{i=1}^{n} h_{i} .
$$

It is a homotopy to zero. Summing over all hypercubes composing the chain complex $B^{\prime}$, the average homotopies described above, one obtains a homotopy to zero for the whole complex $B^{\prime}$. Hence, over $\mathbb{Q}$, there is a canonical choice of homotopies for the applications of Lemma 6 and therefore, a canonically defined differential on the set of Bigelow's generators.

Theorem 1 and Corollary 8 remain true for the odd Khovanov homology [8]. More precisely, the proof only depends on the fact that one can endow enhanced Kauffman's states with a differential that respects the increasing filtration associated to the grading $R$ and such that the part of the differential that respects the grading $R$ is connecting elements in $\mathcal{K} \backslash \mathcal{H}$. The differential constructed in [8] is up to signs the original Khovanov differential. Hence, we also have the following theorem:

Theorem 9 There exists a differential $\delta$ on the free abelian group $B$ generated by Bigelow's generators $\mathcal{G}$, that respects $J$, increases $P$ by 1 and such that the homology of the chain complex $(B, \delta)$ is the odd Khovanov homology. Moreover, over $\mathbb{Q}$, this differential is canonical.

\section{Appendix}

We give the proof of Lemma 6 .

Proof We begin by proving that $(A, a+d h c)$ is a chain complex. Notice that $\delta^{2}=0$ and $b^{2}=0$ imply

$$
c d=0 .
$$


Moreover, $\delta^{2}=0$ also implies

$$
\begin{aligned}
a^{2}+d c & =0, \\
a d+d b & =0, \\
c a+b c & =0 .
\end{aligned}
$$

Hence,

$$
\begin{aligned}
(a+d h c)(a+d h c) & =a^{2}+d h c a+a d h c+d h c d h c \\
& =a^{2}+d h c a+a d h c \\
& =-d c-d h b c-d b h c \\
& =-d(\mathrm{Id}+h b+b h) c \\
& =0 .
\end{aligned}
$$

We define a chain map $f$ from $(C, \delta)$ to $(A, a+d h c)$ by the formula

$$
f=\left(\begin{array}{ll}
1 & d h
\end{array}\right)
$$

and a chain map $g$ from $(A, a+d h c)$ to $(C, \delta)$ by the formula

$$
g=\left(\begin{array}{c}
1 \\
h c
\end{array}\right)
$$

It is straightforward to check that $f \delta=(a+d h c) f$ and $\delta g=g(a+d h c)$ using (9), (12), (11) and $-\mathrm{Id}=h b+b h$. We define a homotopy

$$
H=\left(\begin{array}{ll}
0 & 0 \\
0 & h
\end{array}\right)
$$

going from $C$ to $C$. It follows from (9) and $-\mathrm{Id}=h b+b h$ that $g f-\mathrm{Id}=H \delta+\delta H$. We define $H^{\prime}$ as follows: $H^{\prime}=d h^{3} c$. Let us check that

$$
f g-\mathrm{Id}=H^{\prime}(a+d h c)+(a+d h c) H^{\prime} .
$$

We have

$$
f g-\mathrm{Id}=\mathrm{Id}+d h^{2} c-\mathrm{Id}=d h^{2} c .
$$

Moreover, we have

$$
\begin{aligned}
d h^{2} c & =-d h b h^{2} c-d h^{2} b h c \\
& =2 d h^{2} c+d b h^{3} c+d h^{3} b c \\
& =2 d h^{2} c-a d h^{3} c-d h^{3} c a,
\end{aligned}
$$


from which we deduce

$$
d h^{2} c=d h^{3} c a+a d h^{3} c .
$$

Hence, combining (13) and (14), we obtain

$$
f g-\mathrm{Id}=d h^{3} c a+a d h^{3} c .
$$

Moreover,

$$
\begin{aligned}
H^{\prime}(a+d h c)+(a+d h c) H^{\prime} & =d h^{3} c(a+d h c)+(a+d h c) d h^{3} c \\
& =d h^{3} c a+a d h^{3} c .
\end{aligned}
$$

\section{References}

[1] S Bigelow, A homological definition of the Jones polynomial, from: "Invariants of knots and 3-manifolds (Kyoto, 2001)", (T Ohtsuki, T Kohno, T Le, J Murakami, J Roberts, V Turaev, editors), Geom. Topol. Monogr. 4 (2002) 29-41 MR2002601

[2] I A Dynnikov, Arc-presentations of links. Monotonic simplification arXiv: math.GT/0208153v3

[3] E Grigsby, S Wehrli, On the colored Jones polynomial, sutured Floer homology and knot Floer homology arXiv:math.GT/0807.1432

[4] M Khovanov, A categorification of the Jones polynomial, Duke Math. J. 101 (2000) 359-426 MR1740682

[5] C Manolescu, Nilpotent slices, Hilbert schemes, and the Jones polynomial, Duke Math. J. 132 (2006) 311-369 MR2219260

[6] C Manolescu, P Ozsváth, S Sarkar, A combinatorial description of knot Floer homology, Ann. of Math. (2) 169 (2009) 633-660 MR2480614

[7] C Manolescu, P Ozsváth, Z Szabó, D Thurston, On combinatorial link Floer homology (2007) MR2372850

[8] P Ozsváth, J Rasmussen, Z Szabó, Odd Khovanov homology arXiv: math.GT/0710.4300

[9] P Ozsváth, Z Szabó, On the Heegaard Floer homology of branched double-covers, Adv. Math. 194 (2005) 1-33 MR2141852

[10] J Rasmussen, Knot polynomials and knot homologies, from: "Geometry and topology of manifolds", (H U Boden, I Hambleton, A J Nicas, B D Park, editors), Fields Inst. Commun. 47, Amer. Math. Soc. (2005) 261-280 MR2189938

[11] P Seidel, I Smith, A link invariant from the symplectic geometry of nilpotent slices, Duke Math. J. 134 (2006) 453-514 MR2254624

[12] O Viro, Remarks on definition of Khovanov homology arXiv:math.GT/0202199v1 
Institut für Mathematik, Universität Zürich

Winterthurerstrasse 190, CH-8057 Zürich, Switzerland

Department of Mathematics, University of Aarhus

DK-8000 Aarhus, Denmark

jean-marie.droz@math.uzh.ch, wagner@imf.au.dk

Received: 14 March 2009 Revised: 6 May 2009 